BMJ Open Sport \& Exercise Medicine

\title{
Reduced muscular fatigue after a 12- week leucine-rich amino acid supplementation combined with moderate training in elderly: a randomised, placebo-controlled, double- blind trial
}

Claudia A Reule, ${ }^{1}$ Claudia Scholz, ${ }^{2}$ Christiane Schoen, ${ }^{1}$ Niklas Brown, ${ }^{3}$ Anne Siepelmeyer, ${ }^{1}$ Wilfried W $\mathrm{Alt}^{3}$

\section{ABSTRACT}

Background: Age-related muscle loss is characterised by a progressing decrease in muscle mass, strength and function. Besides resistance training and physical activity, appropriate nutrition that is rich in protein, especially branched-chain amino acids, is very important to support training effects and positively influence the protein synthesis to degradation ratio. Aim: The purpose of this study was to evaluate the effect of a 12-week leucine-rich amino acid supplementation in combination with moderate training.

Methods: Forty-eight healthy subjects exercised for 30 min three times per week and received either a leucine-rich amino acid supplementation or a placebo. Before and after supplementation, volunteers performed an exhaustive eccentric exercise protocol. Maximal concentric strength, muscle soreness, creatine kinase (CK), type II collagen collagenase cleavage neoepitope (C2C), C propeptide of type II procollagen (CP2) and safety assessments were performed before exercise and after 3, 24, 48 and 72 hours.

Results: The supplementation with leucine resulted in reduced loss of strength at 0 and 3 hours after downhill walking compared with the placebo $(p=0.0439)$. The reduction of C2C/CP2 ratio deflection was significantly increased $(p=0.038)$ due to leucine compared with the placebo. The same tendency could be observed for the recovery phase. No significant supplement effects for muscle soreness and CK could be observed.

Conclusion: The principle findings show that leucinerich amino acid supplementation can counteract the negative effects of eccentric exercise. The treatment resulted in a reduction of exercise-induced strength loss.

\section{INTRODUCTION}

Age-related muscle loss is characterised by a progressing decrease in muscle mass,

\section{What are the new findings?}

- The stress test presented here is suitable to evaluate muscular changes and may be used to analyse joint metabolism, even in elderly with healthy joints.

Pronounced training effects could be observed by moderate training with the Nintendo Wil performed three times a week for $30 \mathrm{~min}$ in an elderly population.

- A supplementation with branched-chain amino acid that is rich in leucine can reduce exerciseinduced strength loss and may contribute indirectly to the protection of the joint complex.

strength and function. Beginning in the mid-1930s, 3\%-8\% of the body's skeletal muscle mass may be lost per decade, and this loss rate even accelerates after the age of $60 .^{1}$ This continuous decline in muscle mass is accompanied by impaired mobility and, consequently, decreased physical activity. Impaired mobility has a medical impact, such as increased risk for falls and fractures, increased morbidity, loss of independence, depression and other adverse consequences, and is linked to the loss of quality of life. Interestingly enough, people do not link the loss of mobility to the loss of muscle mass and therefore do not take action for prevention. It is plausible that decreased physical mobility is caused by muscle atrophy and indirectly by decreased joint stability due to sarcopenia in the jointsurrounding muscles. A loss of muscle strength therefore results in a decreased protection of the affected joints from physical stress and a higher risk of joint 
overload and concomitant disturbance of cartilage homeostasis. As cartilage homeostasis is influenced by ageing anyway, ${ }^{2}$ loss of muscular protection could have an additional negative effect on joint health, which could lead to an earlier overturn of balance. The complex process of age-related muscle loss is facilitated by multiple factors, including less activity, missing training and a suboptimal diet."

Although the mechanisms leading to age-related muscle loss are likely numerous, a disproportionate ratio of muscle protein breakdown compared with muscle protein synthesis is believed to have high relevance. There is evidence that an increased availability of protein or amino acids can shift the metabolism towards anabolism and particularly enhance muscle protein synthesis in young and older subjects. ${ }^{4}$ Branched-chain amino acids (BCAAs), leucine in particular, may be responsible for the amino-acidinduced stimulation of muscle protein synthesis. ${ }^{1}$ Leucine appears to play a key role in the regulation of muscle protein synthesis, ${ }^{5}$ and most notable effects on amino -acid metabolism in the skeletal muscle have been observed for leucine. ${ }^{6} 7$ One site for leucine action is a kinase in the insulin signalling cascade previously identified as the mammalian target of rapamycin (mTOR), which has a central role in the regulation of protein synthesis. Leucine also stimulates activation of a second initiation factor eukaryotic initiation factor $4 \mathrm{G}$ through an mTOR-independent kinase. ${ }^{6}$ Additionally, leucine seems to play a role in decreasing the protein degradation rate. ${ }^{5} 68$ Strength training and activity are very important in preventing agerelated muscle loss due to its influence on muscle mass and protein synthesis. ${ }^{1}$ For initiation of muscle protein synthesis and hypertrophy, a complementary, appropriate nutrition that is rich in protein, especially BCAAs, is also important to support training effects and may positively influence the protein synthesis to protein degradation ratio. ${ }^{1}$ The whole-body protein synthesis declines from birth to old age. ${ }^{9}$ High-protein meals have been shown not to enhance the myofibrillar synthesis ${ }^{10}$ but amino acids alone to stimulate muscle protein anabolism in younger and older people ${ }^{11} 12$. As skeletal muscle mass is responsive to changes in physical activity, reduced activity in older people participates to the complex progress of sarcopenia. ${ }^{13}$ If exercise, which positively influences protein homeostasis, is combined with an increased uptake of BCAAs, especially leucine, it could be beneficial in maintaining muscle mass and muscle function and prevent sarcopenia.

Little and Phillips proposed three stimulants of protein synthesis: Leucine, Insulin and resistance training. ${ }^{14}$ A combination of resistance exercise with dietary intake of leucine-enriched amino acids should therefore present the most effective strategy to promote a beneficial relation between protein synthesis and degradation. Due to the role of leucine in the protein synthesis pathway and the muscle degradation mechanism, the focus of interest lies in its influence on muscle building and in its potential for supporting regeneration. The purpose of this study was to evaluate the effect of a 12-week leucine-rich amino acid supplementation in combination with moderate training in elderly participants.

\section{MATERIALS AND METHODS Study design}

The study was a monocentre, randomised, doubleblind, placebo-controlled parallel group study. Prior to trial start, volunteers were informed in detail about the study and signed informed consent before they were screened for eligibility. The nutritional study was approved by the appropriate ethics committee of the Landesäerztekammer Baden-Württemberg, Germany and conducted in orientation to ICHGCP (International Council for Harmonisation's Guideline on Good Clinical Practice) and in compliance with the Declaration of Helsinki. At the beginning and at the end of the 12-week supplementation phase, the volunteers performed an eccentric downhillwalking stress test. Directly before each stress test, immediately after (0 hour) and 3, 24, 48 and 72 hours after the stress test, strength, muscle soreness and biomarker were assessed each. After the first stress test, subjects were randomly assigned to either leucine or the placebo group combined with a special training regimen with Nintendo Wii (Wii Fit Plus and Balance Board). A randomisation scheme was generated using RandList (Tuebingen, Germany). Study products were labelled with the appropriate subject number. Subjects received subject numbers in consecutive order. The study was performed double blindly.

\section{Subjects}

Sample size calculation was calculated by GPower V.3.1.2. The sample size of two independent groups (two tailed) in accordance with the literature, ${ }^{15}$ where the true difference of one repetition maximum in the experimental and control mean was expected to be $16.5 \pm 19 \mathrm{~kg}$, was calculated. Statistical calculation resulted in the inclusion of 22 experimental and 22 control subjects to be able to reject the null hypothesis (power $=0.8$ ). The alpha error level was defined as 0.05 . Considering a dropout rate of 2 volunteers per group, 48 volunteers were enrolled (46 received allocated intervention, and 41 volunteers were analysed), with a 1:1 ratio of men to women (see figure 1).

Five subjects dropped out during the study, as they were not able to perform the stress test the second time due to injury or joint pain. The subjects sustained the injury independent of the study. In addition, no previous injury (eg, osteoarthritis) was known at the moment of inclusion. 
Assessed for eligibility $(n=78)$

\section{Enrolment}

\section{Allocation}

\section{Study completion}

Analysis

Allocated to verum $(n=24)$

Received allocated intervention $(n=24)$

Did not receive allocated intervention $(n=0)$

\section{Excluded - not meeting} inclusion criteria $(n=30)$

\section{Randomized $(n=48)$}

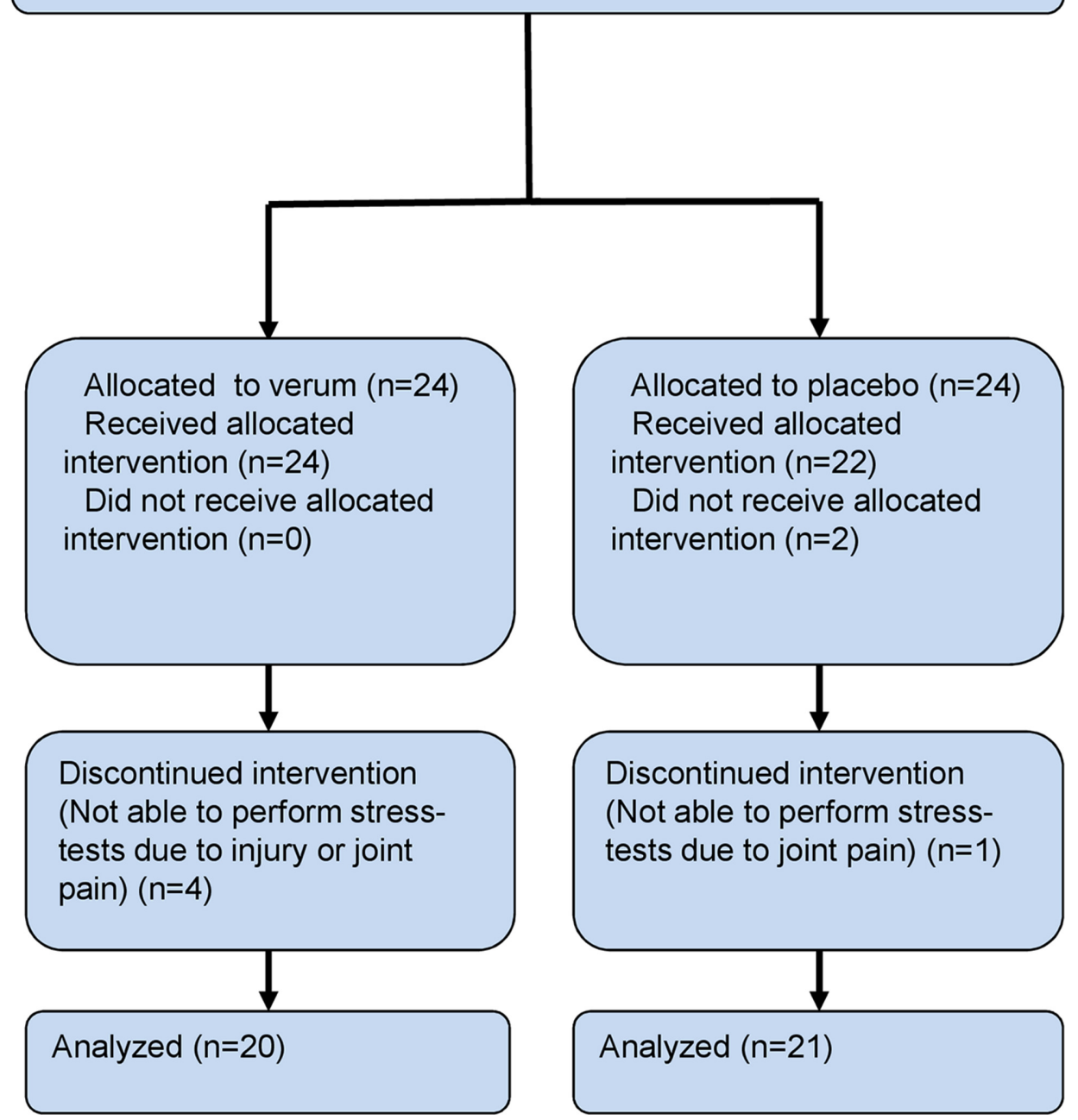

Figure 1 Disposition of volunteers.

Healthy subjects between 55 and 75 years with a body mass index ranging from 19 to $30 \mathrm{~kg} / \mathrm{m}^{2}$, with low activity level and low-to-normal skeletal muscle mass (Skeletal Mass Index, determined by Bioelectrical Impedance Analysis measurement during screening) were included in the current trial (see tables 1 and 2).
Nutritional intervention

Study preparations were consumed once per day, delivered in $200 \mathrm{~mL}$ water prior to the highest activity (directly before the training was started and on a day without training, in the morning prior to leaving the house). On days prior to visits, study compounds had 
Table 1 Inclusion and exclusion criteria for decision of study eligibility

Inclusion criteria

Subject is able and willing to sign the informed consent form prior to screening evaluations.

- Voluntary male and female adults (age 55-75 years)

- BMl: $19-30 \mathrm{~kg} / \mathrm{m}^{2}$

- Non-smoker or smoker with a maximum of 10 cigarettes/ day

- Subject is in good physical and mental health as established by medical history, physical examination, ECG, vital signs, results of biochemistry and haematology.

- Healthy volunteers with borderline-to-normal muscle mass: Definition of cut-off levels as determined by BIA measurement during screening: women SMI $>22 \%$ to $\leq 31 \%$, men $\mathrm{SMI} \leq 31 \%$ to $\leq 40,5 \%$.

- Volunteers have to be non-active athletes. Amateur or professional athletes will not be included in this study.

- Willing to perform accompanied moderate training according to recommendations from WHO for healthy volunteers during the course of the study.

- Not anticipating any planned changes in lifestyle regarding activity and nutrition for the duration of the study

\section{Exclusion criteria}

- Women: known pregnancy, breastfeeding or intention to become pregnant during the study

- Volunteers with high-protein diet (>1-2 $\mathrm{g} / \mathrm{kg}$ body weight) or additional intake of protein supplements/protein-containing bars or shakes

- Relevant history or presence of any medical disorder, potentially interfering with this study (eg, volunteers having experienced any cardiovascular events such as infarction, angina, surgical endocoronary intervention or stroke in the last 6 months or volunteers suffering from diabetes)

- Intake of medications that directly affect parameters of muscle or joint function or inflammation within 2 weeks prior to visit 1 or during the study

- For this study, clinically relevant abnormal laboratory, ECG, vital signs or physical findings at screening

- Known hypersensitivity to the study preparation or to single ingredients

- Regular intake of dietary supplements affecting muscle or joint function within 2 weeks prior to visit 1 or during the study

- Drug, alcohol and medication abuses

- Known HIV infection

- Known acute or chronic hepatitis B and C infection

- Participation in another clinical study within the last 4 weeks and concurrent participation in another clinical study

- Blood donation within 4 weeks prior to study start (visit 1) or during the study

BIA, Bioelectrical Impedance Analysis; BMI, Body Mass Index; SMI, Skeletal Mass Index.

to be taken 24 hours before the appointment at the study site. During visits, the study preparations were ingested immediately before the stress test. The verum preparation consisted of $3.2 \mathrm{~g}$ L-leucine, $0.9 \mathrm{~g} \mathrm{~L}$ valine, $0.9 \mathrm{~g}$ L-isoleucine. The placebo equated to the verum preparation merely without active ingredients.

\section{Training intervention}

Training was carried out according to WHO recommendations for healthy individuals (three times a week, 30 min each) during the course of the trial and was started after the initial stress test. To ensure standardisation and control of the study, the subjects were instructed to use the Nintendo Wii (Wii Fit Plus and Balance Board) for their training. Each training session was divided into three $10 \mathrm{~min}$ sections: aerobic, strength and balance. Table 3 summarises the training routine, which was changed every month. Volunteers received a training plan with detailed information, including training exercises, duration and intensity. Training intensity was increased weekly (see table 3). One week prior to each visit, volunteers were instructed

Table 2 Anthropometric data for men and women allocated to the placebo and the leucine groups

\begin{tabular}{lllll}
\hline Group & $\begin{array}{l}\text { Sample } \\
(\mathbf{n})\end{array}$ & $\begin{array}{l}\text { Age } \\
\text { (years) }\end{array}$ & $\begin{array}{l}\mathbf{B M I} \\
\mathbf{( k g / \mathbf { m } ^ { 2 }}\end{array}$ & $\begin{array}{l}\text { SMI } \\
\text { (\%) }\end{array}$ \\
\hline Placebo & Men (12) & $60 \pm 4$ & $26.7 \pm 1.6$ & $35.7 \pm 3.0$ \\
& Women (12) & $63 \pm 6$ & $26.2 \pm 3.4$ & $25.5 \pm 2.5$ \\
Leucine & Men (12) & $61 \pm 4$ & $25.8 \pm 2.1$ & $34.4 \pm 2.5$ \\
& Women (12) & $66 \pm 6$ & $24.4 \pm 2.0$ & $26.3 \pm 2.4$ \\
\hline
\end{tabular}

BMI, body mass index; SMI, Skeletal Mass Index. 
Table 3 Training routine

\begin{tabular}{|c|c|c|c|}
\hline & Week 1-4 & Weeks 5-8 & Weeks 9-12 \\
\hline \multirow[t]{3}{*}{ Aerobic } & Basis step (2-3 min) & Step super (4-5 min) & Step super (4-5 min) \\
\hline & Hula hoop (2 min) & Obstacle course (2-3 min) & Obstacle course (2-3 min) \\
\hline & Jogging plus (5-6 min) & Rythm kung-fu (2-3 min) & Jogging plus (2-4 min) \\
\hline \multirow[t]{6}{*}{ Strength } & Lunges (2 min) & Rowing (1 min) & Rowing (1 min) \\
\hline & Side steps (2 min) & Lunges (2 min) & Lunges (2 min) \\
\hline & Single-leg twist (1 min) & Single-leg extension (1 min) & Single-leg extension (1 min) \\
\hline & Arm and leg lift (1 min) & Torso twist (3 min) & Torso twist (2 min) \\
\hline & Sideways leg lift $1 \mathrm{~min}$ ) & Sideways leg lift (1 min) & Lunges (2 min) \\
\hline & Torso twist (3 min) & Push-ups (2 min) & Push-ups (2 min) \\
\hline \multirow[t]{7}{*}{ Balance (five games out of seven) } & & Soccer heading (2 min) & \\
\hline & & Ski slalom (2 min) & \\
\hline & & Ski jump (2 min) & \\
\hline & & Balance bubble (2 min) & \\
\hline & & Tightrope walk (2 min) & \\
\hline & & Penguin slide (2 min) & \\
\hline & & Snowboard slalom (2 min) & \\
\hline
\end{tabular}

not to exercise with the Nintendo Wii to avoid influences of training on performance during the stress test and further ensure capturing of parameters under the resting state. The training was controlled regularly by extracting the training protocols recorded with the Nintendo Wii.

\section{Stress test}

For the evaluation of product-specific effects on muscular fatigue, volunteers had to perform eccentric downhill walking $(25 \%$ gradient on a treadmill (Woodway Ergo XERG90 specifications) with an additional load of $10 \%$ body weight (weight vest). Women had to walk at $4 \mathrm{~km} /$ hour, and men had to walk at $5 \mathrm{~km} /$ hour. To evaluate the individual performance level, volunteers were asked to walk as long as possible without exceeding a maximum of $40 \mathrm{~min}$ at the initial stress test. For the second stress test, the individual protocol from the first stress test was repeated to ensure a comparable physical load for each volunteer. Within the clinical study, a set of up-to-date methods was used, involving analyses of pain, muscle strength and cartilage, inflammation and muscle degeneration markers.

\section{Strength assessment}

Muscle strength was measured at each point of time by determining the mean out of two isometric maximal voluntary contractions (MVCs) of the left quadriceps femoris muscle. To determine MVCs, a leg extension device was used (David Health Solution, Helsinki,
Finland). Each measurement was conducted two times, and the mean value was used. The strength measurement was accomplished by well-trained, strengthmeasurement-experienced staff and under standardised conditions. The subjects were instructed to gradually contract their muscle to a maximum force during a three-step command. The observer pushed the volunteer verbally until the strength measurement system did not show any augmentation. Usually, the contraction time takes about $4-5 \mathrm{~s}$. With regard to the older collective, no electromyostimulation was used to control for MVC due to ethical aspects.

\section{Muscle soreness}

Volunteers were asked to rate their global muscular pain (muscle soreness) on a $10 \mathrm{~cm}$ visual analogue scale. Volunteers rated their muscle soreness before and at each time point after the stress test $(0,3,24,48$ and 72 hours).

\section{Biomarkers}

The test battery included blood sampling to determine serum creatine kinase $(\mathrm{CK})$ as a marker for muscle degradation and C-propeptide of type II procollagen (CP2) and type II collagen collagenase cleavage neoepitope (C2C) to detect potential cartilage synthesis or degradation. All biomarkers were assessed at each point of time (pre stress test, 0, 3, 24, 48 and 72 hours after stress test). Aliquots of serum were taken after centrifugation $\left(3000 \mathrm{~g}, 4^{\circ} \mathrm{C}, 10 \mathrm{~min}\right) 30 \mathrm{~min}$ after blood donation. All samples were analysed batchwise for each 
subject at the end of the study to avoid interassay variations. CK was determined in the routine laboratory (Synlab, Leinfelden-Echterdingen, Germany). To determine CP2 and C2C, a competitive immune assay was used (Ibex Pharmaceutical, Montreal, Canada). The ratio of C2C and CP2 values of $<1$ indicates higher cartilage synthesis compared with cartilage degradation. However, values of $>1$ reflect predominance of cartilage degradation. The main focus of the study was to observe the influence of the intervention on muscle function and muscle degeneration. The observation of cartilage homeostasis was an incidental question. Therefore, a representative cartilage degradation and synthetis marker was measured. The C2C/ $\mathrm{CP} 2$ ratio gives a good overview of the homeostasis, as it includes cartilage degradation and synthesis.

\section{Safety assessments}

To assess the safety of the study products, vital signs and blood samples for routine laboratory were determined at all visits. Additionally, adverse events and concomitant medication were documented.

\section{Statistics}

Analysis and statistics were done using GraphPad Prism V.5.0 (SPSS and Microsoft Excel). For strength assessment, different periods after the stress test were defined: 0-3 hours was defined as an acute phase, and 24-72 hours was defined as a regeneration phase. To quantify the acute effects before and after intervention, a delta of preintervention to 0 hour and a delta of preintervention to 3 hours was averaged for each group and visit. Out of these acute-strength reductions, a delta of postintervention to preintervention was calculated. The same was done for the regeneration phase time points. An unpaired t-test (differences between groups) on individual parameters was used for statistical analysis if parameters were Gaussian distributed (Shapiro-Wilk test). A Mann-Whitney U test was used in case of non-normal distributions. The same analysis between groups was done for the $\mathrm{C} 2 \mathrm{C} / \mathrm{CP} 2$ ratio. For changes within a group, one-way repeated measures analyses of variance (rmANOVAs) were assessed. In the case of CK values, extreme outliers were observed. Therefore, data were log transformed prior to analysis. For CK and Visual Analogue Scale, area under the curve (AUC) was calculated and compared between groups using an unpaired t-test or a Mann-Whitney $\mathrm{U}$ test, if appropriate. For the $\mathrm{C} 2 \mathrm{C} / \mathrm{CP} 2$ ratio, the values were normalised to the pretreatment time point.

\section{RESULTS}

\section{Strength assessment}

Before, immediately after and 3, 24, 48 and 72 hours after the stress test, MVC of quadriceps femoris muscle of the left leg was assessed to document muscular strength loss induced by downhill walking. Baseline conditions before and after treatment were not
Strength loss - Baseline corrected Placebo

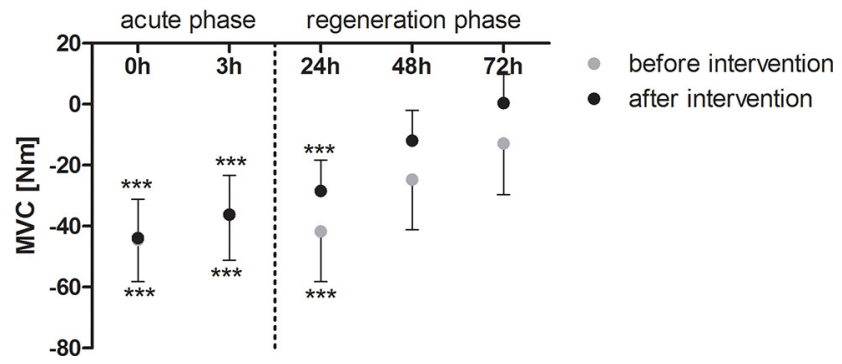

Figure 2 Baseline corrected strength loss after the eccentric stress test for the placebo group (mean $\pm 95 \%$ $\mathrm{Cl})$. h, hours; MVC, maximal voluntary

contraction. ${ }^{\star \star \star} \mathrm{p}<0.001$.

significantly different, neither for the placebo nor for the leucine group, indicating the absence of a training effect in MVC due to intervention.

After the stress test, a clear strength reduction induced by downhill walking could be observed, which was true for for the stress test before and after intervention and visible in the placebo and the leucine groups (rmANOVA; $\mathrm{p}<0.0001$ each). In particular, immediately after and at 3 and 24 hours after the stress test, strength was significantly reduced due to the stress test. In figure 2 and figure 3, the baseline corrected knee extension strength at each time point is depicted to show strength reduction due to downhill walking. In particular, in the acute phase ( $0-3$ hours), the regimen leucine plus training was able to buffer the strength loss, induced by downhill walking. Figure 4 shows the averaged differences (visit 4 compared with visit 1) of the baseline corrected time points 0-3 hours and 2448 hours. This depicts the extent of strength reduction due to intervention for the acute phase after the stress test and the extent of strength reduction for the recovery phase after the stress test. The administration of leucine, plus training, resulted in a reduced loss of strength at 0-3 hours (acute phase) after compared with placebo plus training $(\mathrm{p}=0.0439$; figure $4)$. For the regeneration phase (24-48hours), the

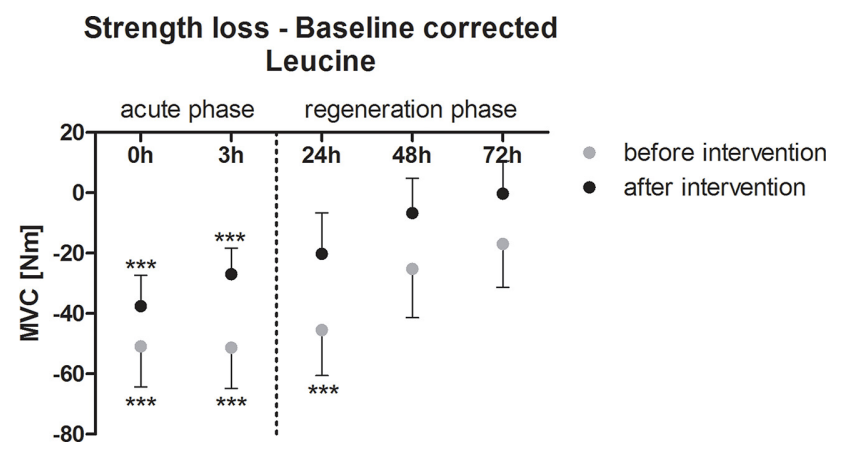

Figure 3 Baseline corrected strength loss after eccentric stress test for the leucine group (mean $\pm 95 \% \mathrm{Cl}$ ). $\mathrm{h}$, hours; MVC, maximal voluntary contraction. ${ }^{\star \star *} \mathrm{p}<0.001$. 


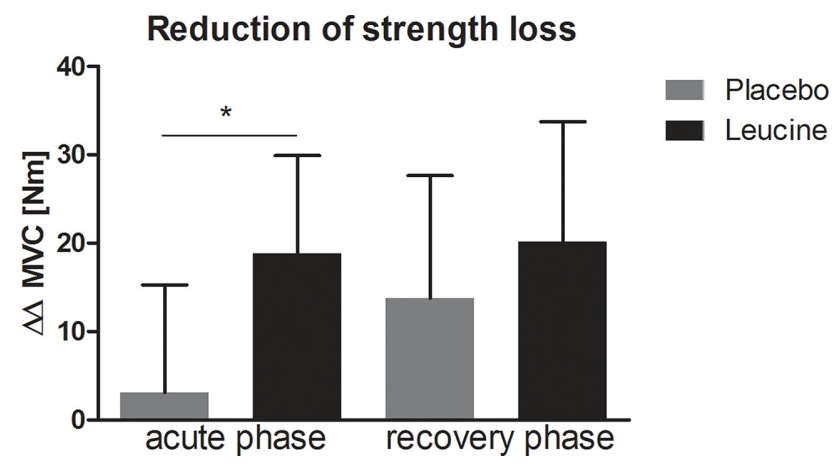

Figure 4 Reduction of strength loss after intervention described as averaged differences (visit 4 compared with visit 1) of the baseline corrected time points Ohours and 2448 hours. This depicts the extent of strength reduction due to intervention for the acute phase after the stress test and the extent of strength reduction for the recovery phase after the stress test (mean+95\% Cl). MVC, maximal voluntary contraction. ${ }^{*} \mathrm{p}<0.05$.

difference between leucine and placebo was not significant $(\mathrm{p}=0.2134)$.

\section{Muscle soreness}

Participants reported muscle soreness after the stress test. Figure 5 and figure 6 show the significant increase in muscle soreness in both groups during the regeneration phase before and after intervention $(p<0.0001$, table 4 ). The maximum of muscle soreness could be observed at 48 hours after the stress test. After 72 hours, the pain did not return to baseline. In the placebo and the leucine groups, training effects were observed. Muscle soreness was reduced after intervention. However, no treatment effects could be observed. AUC was not significantly different between groups $(\mathrm{p}=0.5798$, figure 7$)$.

\section{Biomarkers}

Before and after intervention, CK levels were markedly elevated due to downhill walking (see table 4),

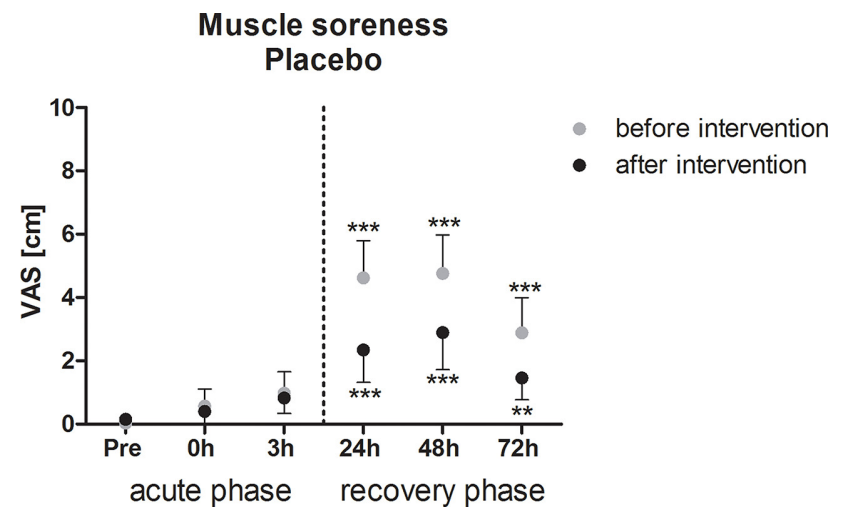

Figure 5 Muscle soreness after eccentric stress test for the placebo group (mean $\pm 95 \% \mathrm{Cl}$ ). $\mathrm{h}$, hours; Pre, preintervention; VAS, Visual Analogue Scale. ${ }^{* \star *} \mathrm{p}<0.001,{ }^{\star \star} \mathrm{p}<0.01$.
Muscle soreness Leucine

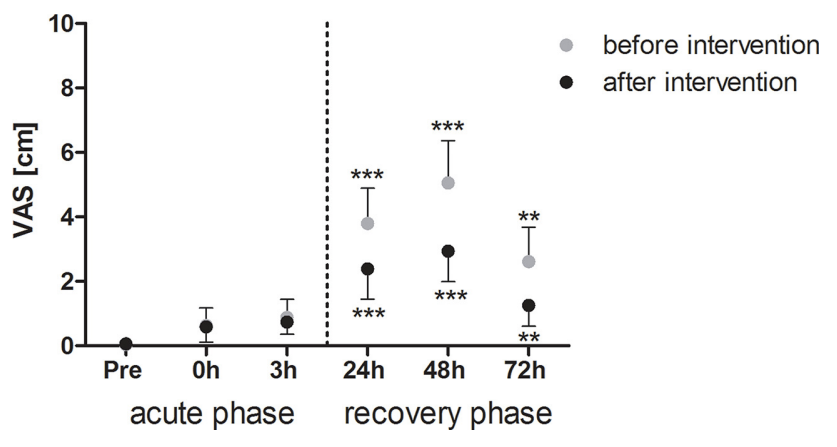

Figure 6 Muscle soreness after eccentric stress test for the leucine group (mean $\pm 95 \% \mathrm{Cl}$ ). h, hours; Pre, preintervention; VAS, Visual Analogue Scale. ${ }^{\star \star \star} p<0.001,{ }^{\star \star} p<0.01$.

indicating increased muscle degeneration processes. After intervention, CK was clearly reduced in the placebo and the leucine groups compared with CK levels before intervention (table 4). However, no difference in CK reduction (AUC) between intervention groups could be observed.

After the stress test, the $\mathrm{C} 2 \mathrm{C} / \mathrm{CP} 2$ ratio declined significantly over time in the placebo and the leucine groups before and after intervention (placebo before intervention: $p=0.002$; placebo after intervention: $p<0.001$; leucine before intervention: $p=0.0436$; leucine after intervention; $p=0.0403$; table 4 ). In the placebo group, after intervention, the $\mathrm{C} 2 \mathrm{C} / \mathrm{CP} 2$ ratio was deflected more compared with baseline. In the leucine group, it was vice versa. A decreased deflection could be observed after intervention. To investigate differences between groups, the acute phase and the regeneration phase were analysed separately (figure 8 ). In the acute phase, the reduction of $\mathrm{C} 2 \mathrm{C} / \mathrm{CP} 2$ ratio

\section{Reduction of muscle soreness}

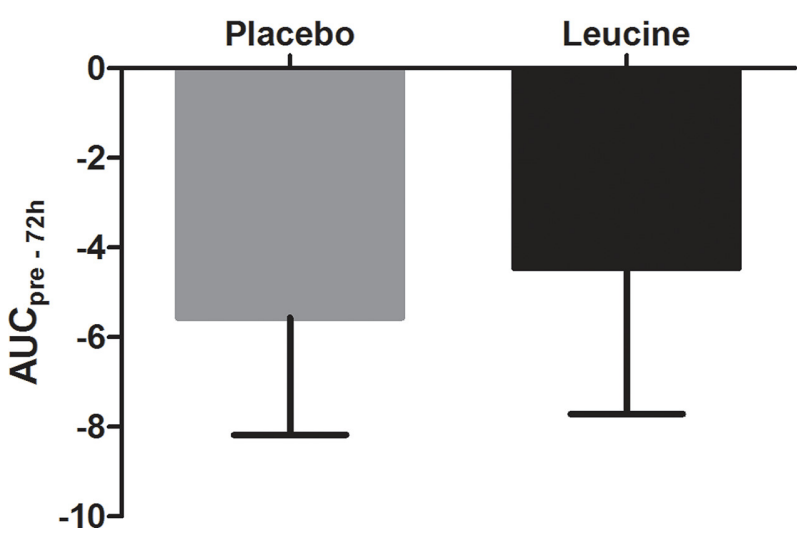

Figure 7 Reduction of muscle soreness after intervention, described as AUC of the difference after intervention minus before intervention for placebo and leucine (mean $\pm 95 \%$ $\mathrm{Cl})$. AUC, area under the curve, pre-72 h, preintervention to 72 hours. 


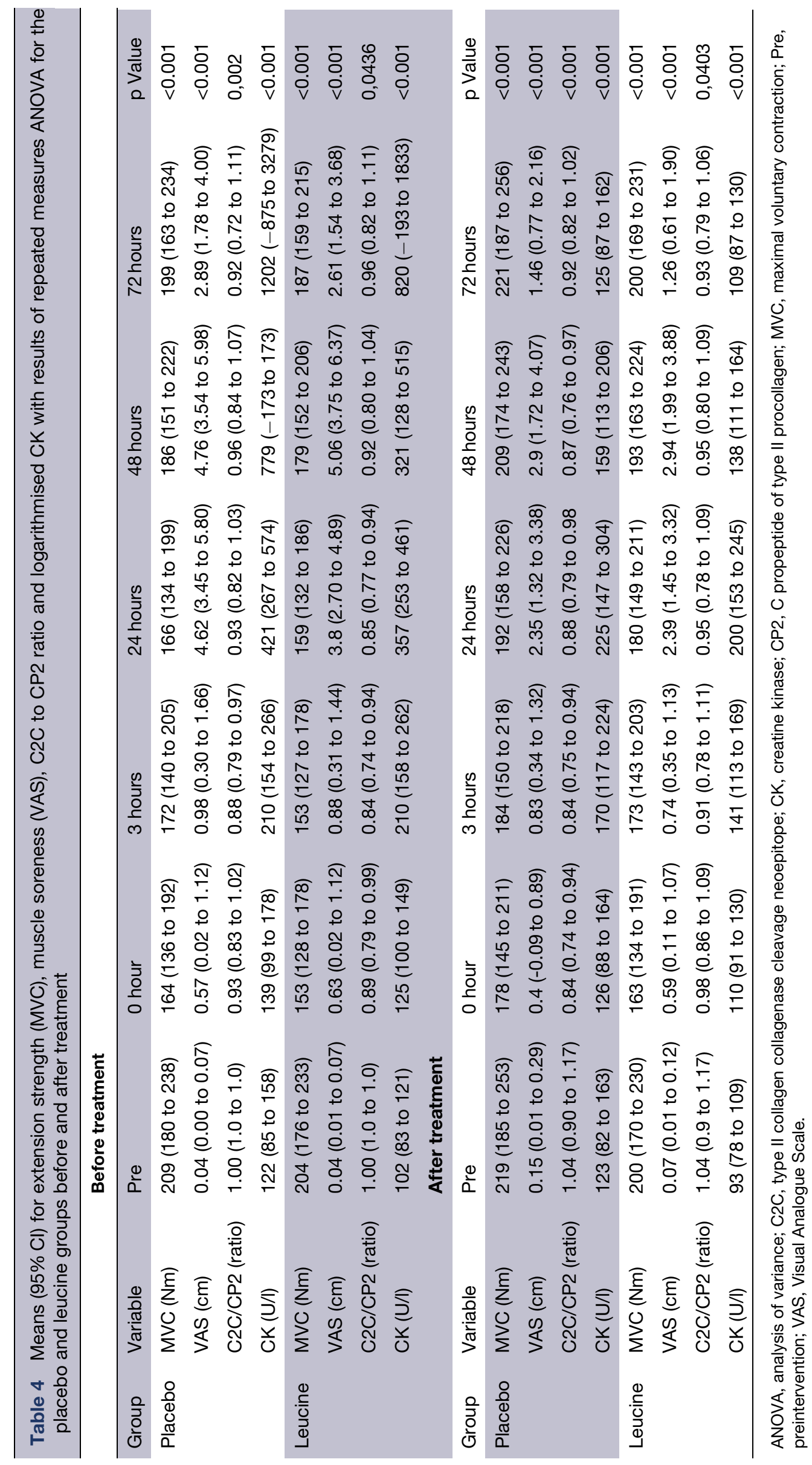


Reduction of $\mathrm{C} 2 \mathrm{C} / \mathrm{CP} 2$ deflection

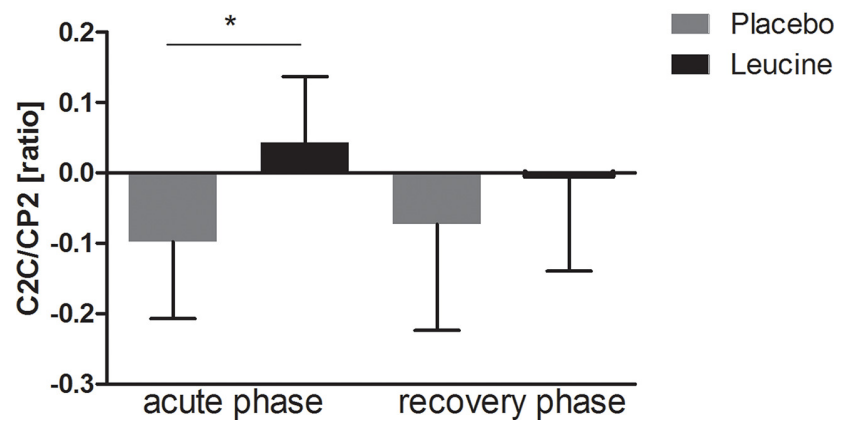

Figure 8 Reduction of C2C/CP2 deflection after intervention (mean+95\% Cl). C2C, type II collagen collagenase cleavage neoepitope; CP2, C propeptide of type II procollagen. ${ }^{*} \mathrm{p}<0.05$.

deflection was significantly increased $(\mathrm{p}=0.038)$ due to leucine compared with the placebo. In the regeneration phase, the difference between the placebo and leucine was not significant but showed the same tendency.

\section{DISCUSSION}

The aim of the current study was to examine the effects of a 12-week leucine-rich amino acid supplementation in combination with moderate training in untrained, healthy elderly. The principal findings show that a leucine-rich amino acid supplementation can counteract the negative effects of eccentric exercise. The treatment with leucine-rich amino acids resulted in a reduction of exercise-induced strength loss during the acute phase of regeneration.

\section{Effects of eccentric downhill-walking exercise}

The currently used exercise protocol successfully induced muscle damage, shown by a significant strength reduction, appearance of muscle soreness, increase of $\mathrm{CK}$ levels and deflection of cartilage homeostasis $(\mathrm{C} 2 \mathrm{C} / \mathrm{CP} 2$ ratio). The $\mathrm{C} 2 \mathrm{C} / \mathrm{CP} 2$ ratio represents the effects of downhill walking on cartilage homeostasis. This is of interest to clarify whether changes in strength loss after downhill walking could also influence the loading of joints. This supports the efficacy of loaded downhill walking as a model to induce muscle damage in an untrained, healthy collective of elderly. The model represents a realistic load model close to the everyday life of healthy untrained elderly with normal mobility. The degree of muscle degeneration was comparably high. None of the assessed parameters returned to baseline after 72 hours, which was previously observed in several studies but, in most cases, in a trained collective. ${ }^{16}$

In addition to the induced muscle damage, downhill walking clearly disturbed cartilage homeostasis in a healthy collective without joint impairment. It can be hypothesised that due to muscular fatigue, the muscular protection of joints might have been reduced, and the mechanical impact of downhill walking could be directly transferred to the joint complex. In a normal healthy situation, cartilage synthesis and degradation are in balance. If degradation processes are increased, for example, due to high mechanical load, compensatory mechanisms are upregulating synthesis processes to retrieve homeostasis. ${ }^{17}$ Interestingly, the load induced by downhill walking in the current study triggered the cartilage synthesis to a higher extent than the cartilage degradation. This is in line with the literature for a healthy situation to achieve a steady state. ${ }^{17} 18$ The stress test used in this study seems to also be suitable to investigate changes of joint metabolism in a healthy situation.

\section{Training effects}

In the current study, moderate training was either combined with a leucine-enriched amino acid treatment or a placebo treatment. As expected, training effects could be documented in both intervention groups. As study products supporting muscle mass or muscle structure are supposed to be mostly beneficial in combination with training, it might be challenging to demonstrate the superiority of the product compared with a placebo. In particular, in an untrained collective, training effects may overlay the supplement effects. The majority of parameters were clearly improved after intervention: muscle soreness after intervention was much lower in both groups, also reflected by decreased muscle degeneration (CK levels). It is worth mentioning that these clear training effects were achieved by training with only moderate intensity, three times a week for $30 \mathrm{~min}$, including aerobic, strength and balance training.

\section{Supplement effects}

The strength loss caused by muscular fatigue was less pronounced after 3 months of supplementation in the treatment group compared with a placebo. The reduction of strength loss was significant between groups for the acute phase within the first 3 hours after downhill walking. This means that acute strength loss induced by the stress protocol was lower after intervention in the treatment group compared with the placebo. It was hypothesised that a better muscular support could protect the joints against overload due to better active joint stability. To evaluate if reduced strength loss after exercise could also protect the joint complex, biomarkers for cartilage homeostasis were determined during the current study. During the current study, a significant lower acute deflection of the $\mathrm{C} 2 \mathrm{C} / \mathrm{CP} 2$ ratio could be found in the treatment group compared with the placebo. The lower deflection of $\mathrm{C} 2 \mathrm{C} / \mathrm{CP} 2$ could be interpreted as a lower disturbance of joint homeostasis and a reflection of a higher level of steady state.

Even if it is speculative, it can be assumed that as a consequence of reduced strength loss in the leucine group, the load of the joint complex was reduced, 
which was supported by less disturbance of the $\mathrm{C} 2 \mathrm{C} /$ CP2 ratio.

Conversely, for muscle soreness and CK levels, no supplementation-specific effects could be observed. This is in conflict with previously published data, where BCAA supplementation including leucine showed effectiveness for muscle strength, muscle soreness and CK levels. ${ }^{16}$ However, in that study, the acute reactions were not measured. Furthermore, the collective consisted of resistance-trained young men, with resistance training twice a week. They proceeded with their normal training during the study without additional training. Therefore, it is likely that training effects were lower in the study of Howatson ${ }^{13}$ compared with the untrained collective of the current study. Usually, in an untrained collective, even moderate basic training results in high training effects in the first weeks. Presumably, these training effects covered supplement effects for muscle soreness and CK in the presented study.

Strength loss after exercise seems to be less affected by the moderate training. A possible explanation might be the low training intensity of the performed training using the Nintendo Wii. In this case, a higher intensity can only be achieved by a higher repetition number but not with a higher training load. Therefore, the training effects in the current study were predominantly in the area of basic training and strength endurance.

However, functional impairment by eccentric downhillwalking exercise is better tolerated if moderate training is combined with a leucine-enriched amino acid supplementation. Leucine is involved in protein synthesis by influencing the mTOR pathway ${ }^{19}$ and in decreased protein degradation. ${ }^{20}$ The effectiveness of BCAA on the reduction of muscle damage has been shown previously. ${ }^{16}$ The results presented here demonstrate the efficacy of leucine-rich amino acid supplementation in untrained elderly. The combination with moderate training was sufficient to trigger muscle metabolism in such a way that muscle fatigue was less pronounced after eccentric exercise. In addition, the reduction of strength loss may contribute to a better muscular protection of the loaded joint complex. A supplement rich in leucine, combined with moderate training, seems to be a potential intervention in preventing joint overload and muscular fatigue due to eccentric exercise in an older collective. Further studies should clarify if such an intervention could be suitable to prevent from sarcopenia.

Acknowledgement We would like to thank all volunteers who took part in this study.

Contributors CAR, CSchoen, NB, AS and WWA were responsible for study planning, study realisation, data analysis and reporting. CScholz contributed to discussions about the study design prior to the start of the study. CAR wrote the first manuscript, and all other authors critically reviewed the manuscript and contributed with suggestions.

Funding The study was sponsored by Seven Seas. Seven Seas provided the food supplement (study medication) used in the study.
Competing interests The sponsor contributed to discussion about study design and selection of outcome measures prior to study start. During the measurements and data analysis, all data were completely blinded.

Measurements and data analysis were executed by CRO BioTeSys $\mathrm{GmbH}$ and the Department of Sport and Exercise Science of the University of Stuttgart.

Ethics approval Ethical approval was obtained from the ethical committee of the Landesärztekammer Baden-Württemberg prior to the study of the start.

Provenance and peer review Not commissioned; externally peer reviewed.

Open Access This is an Open Access article distributed in accordance with the Creative Commons Attribution Non Commercial (CC BY-NC 4.0) license, which permits others to distribute, remix, adapt, build upon this work noncommercially, and license their derivative works on different terms, provided the original work is properly cited and the use is non-commercial. See: http:// creativecommons.org/licenses/by-nc/4.0/

(C) Article author(s) (or their employer(s) unless otherwise stated in the text of the article) 2017. All rights reserved. No commercial use is permitted unless otherwise expressly granted.

\section{REFERENCES}

1. Dreyer HC, Volpi E. Role of protein and amino acids in the pathophysiology and treatment of sarcopenia. J Am Coll Nutr 2005;24:S140-5.

2. Reginster J.-Y, Pelletier J.-P, J.Martel Pelletier, et al. Osteoarthritis: Clinical and Experimental Aspects. Heidelberg: Springer -Verlag, 1999.

3. Paddon-Jones D, Short KR, Campbell WW, et al. Role of dietary protein in the Sarcopenia of aging. Am J Clin Nutr 2008;87:1562S-6.

4. Timmerman KL, Volpi E. Amino acid metabolism and regulatory effects in aging. Curr Opin Clin Nutr Metab Care 2008;11:45-9.

5. Norton LE, Layman DK. Leucine regulates translation initiation of protein synthesis in skeletal muscle after exercise. J Nutr 2006;136:533S-7.

6. Layman DK. Role of leucine in protein metabolism during exercise and recovery. Can J Appl Physiol 2002;27:646-63.

7. Rennie MJ, Tipton KD. Protein and amino acid metabolism during and after exercise and the effects of nutrition. Annu Rev Nutr 2000;20:457-83.

8. Ispoglou T, King RF, Polman RC, et al. Daily L-leucine supplementation in novice trainees during a 12-week weight training program. Int J Sports Physiol Perform 2011;6:38-50.

9. Frontera WR, Meredith $\mathrm{CN}$, O'Reilly KP, et al. Strength training and determinants of VO2max in older men. J Appl Physiol 1990;68:329-33.

10. Yarasheski KE, Zachwieja JJ, Bier DM. Acute effects of resistance exercise on muscle protein synthesis rate in young and elderly men and women. Am J Physiol 1993;265(2 Pt 1):E210-E214.

11. Welle S, Thornton CA. High-protein meals do not enhance myofibrillar synthesis after resistance exercise in 62- to 75-yr-old men and women. Am J Physiol 1998;274(4 Pt 1):E677-E683.

12. Fukagawa NK, Minaker KL, Young VR, et al. Leucine metabolism in aging humans: effect of insulin and substrate availability. Am J Physiol 1989;256(2 Pt 1):E288-E294.

13. Bross $R$, Javanbakht $M$, Bhasin $S$. Anabolic interventions for agingassociated Sarcopenia. J Clin Endocrinol Metab 1999;84:3420-30.

14. Little JP, Phillips SM. Resistance exercise and nutrition to counteract muscle wasting. Appl Physiol Nutr Metab 2009;34:817-28.

15. Børsheim E, Bui QU, Tissier S, et al. Effect of amino acid supplementation on muscle mass, strength and physical function in elderly. Clin Nutr 2008;27:189-95.

16. Howatson G, Hoad M, Goodall S, et al. Exercise-induced muscle damage is reduced in resistance-trained males by branched chain amino acids: a randomised, double-blind, placebo controlled study. J Int Soc Sports Nutr 2012;9:20.

17. Andriacchi TP, Koo S, Scanlan SF. Gait mechanics influence healthy cartilage morphology and osteoarthritis of the knee. J Bone Joint Surg Am 2009;91(Suppl 1):95-101.

18. Leumann A, Horisberger M, Valderrabano V. Mechano-biologische verknüpfung the joint as an organ-Konzept. Schweizerische Zeitung für Sportmedizin und Sporttraumatologie 2011;59:161-4.

19. Little JP, Phillips SM. Resistance exercise and nutrition to counteract muscle wasting. Appl Physiol Nutr Metab 2009;34:817-28.

20. da Luz CR, Nicastro $\mathrm{H}$, Zanchi NE, et al. Potential therapeutic effects of branched-chain amino acids supplementation on resistance exercise-based muscle damage in humans. J Int Soc Sports Nutr 2011;8:23-8. 